Research Article

\title{
Electromagnetic Interference between Cranes and Broadcasting Antennas
}

\author{
V. Javor \\ Faculty of Electronic Engineering, University of Nis, A. Medvedeva 14, 18000 Nis, Serbia \\ Correspondence should be addressed to V. Javor; vesna.javor@elfak.ni.ac.rs
}

Received 22 July 2015; Accepted 18 October 2015

Academic Editor: Felipe Cátedra

Copyright (C) 2015 V. Javor. This is an open access article distributed under the Creative Commons Attribution License, which permits unrestricted use, distribution, and reproduction in any medium, provided the original work is properly cited.

\begin{abstract}
An interesting phenomenon was noticed in some cases by workers operating with cranes in electromagnetic field of broadcasting antennas. They experienced electrical shock or burning pain at distances of kilometers away from powerful transmitters because cranes act as unintentional receiving antennas. The solution to this problem depends on dimensions and positioning of the crane structure, electromagnetic field strength at the site, frequency, and directional characteristic of the transmitting antenna. Electromagnetic interference between such structures is analyzed in this paper. Computational results for the induced currents and voltages in crane, treated as wire antenna problem, are determined using integrodifferential equations for the current along conductive structure, satisfying boundary condition for the electric field. Point matching method as the Method of Moments (MoM) is applied for solving these equations and polynomial approximation of the current is used. Results are presented for different crane structures and possible solutions to this problem are given.
\end{abstract}

\section{Introduction}

When considering interference between natural electromagnetic sources of disturbances (terrestrial, as lightning discharges, or extraterrestrial, as cosmic radiation and solar wind) and various man-made objects (such as equipment, electronic devices, electric systems, power lines, communication and automation systems, and installations), the key issues are electromagnetic field strength, amplitudes of voltages and currents, electrical properties of the medium, dimensions and positioning of the structure, distances, and frequency. An adequate modeling would take into account a wide variety of configurations of the observed systems. In the last few decades rapid advance has been achieved due to development of numerical programs and modern computers possibilities. The analysis in this paper is based on the wire antenna approach and the ground is treated as perfectly conducting. Finite ground conductivity has some influence on induced voltages and currents, so it could be considered using the same procedure as in this paper, but the solution to this problem is what matters. However, these electromagnetic interference (EMI) problems were rarely noticed and described just in a few papers [1-7]. Large conductive structures permanently or temporarily positioned in electromagnetic field of powerful medium wave (MW) transmitters, if their dimensions are of the order of wavelength, might be dangerous for humans and equipment. AM transmission is nowadays mostly used for sports, news, and talk radio, whereas FM is better for sound. Digital audio broadcasting is advancing in many countries all over the world. Although there is a shift to higher frequencies for all systems, MW transmitters are still in operation throughout the world. AM carrier frequencies are assigned to stations in the frequency range from 531 to $1611 \mathrm{kHz}$, and, just in Europe, there are still more than a hundred of them with power ranging from $1 \mathrm{~kW}$ to $1 \mathrm{MW}$ [8], depending on the targeted area (local, regional, or international).

\section{Some EMI Problems}

The lifting crane of height $60 \mathrm{~m}$ and jib of length about $25 \mathrm{~m}$ was operating in Progar (Serbia), $8 \mathrm{~km}$ away from the transmitter, when the problem was noticed by crane workers. The measured open-circuit voltage was $450 \mathrm{~V}$. Radio Belgrade was operating at the frequency $f=684 \mathrm{kHz}$ up to 1999 , having powerful $2 \mathrm{MW}$ transmitter and main antenna of height $h=$ 


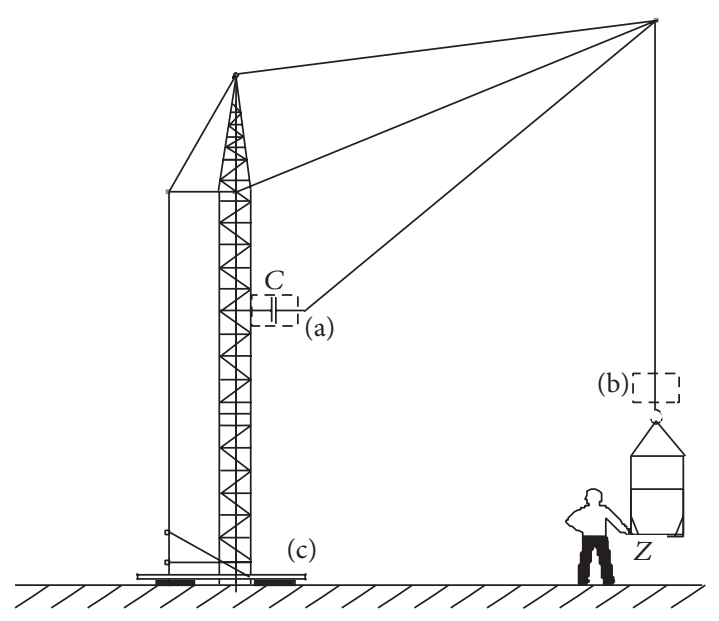

Figure 1: Possible places (a), (b), and (c) for inserting impedance into the crane contour.

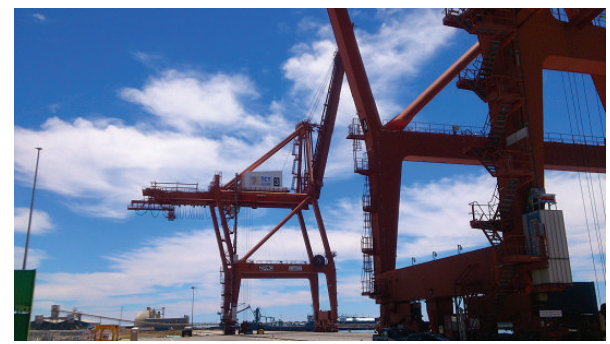

FIGURE 2: Rail-mounted $65 \mathrm{t}$ crane in Tenerife (Spain).

$230 \mathrm{~m}$ (half-wave monopole). The electric field strength was $E_{0}=2.1 \mathrm{~V} / \mathrm{m}$ at the crane's site. The solution to that problem was an egg-insulator inserted above the hook (Figure 1), thus disturbing the resonance of the formed contour. The limitation of this solution is its mechanical strength.

Values of the induced voltages were measured in some similar situations throughout the world. There are railmounted container cranes in the harbor of Tenerife, Spain, having $30.5 \mathrm{~m}$ span and capacity of $65 \mathrm{t}$. At one of them, the industrial bus was faulty each time when it was in the position to take the load from ship, or if its spreader descended near containers. Sensors and actuators were grounded to the crane structure, but the problem in control system persisted. In 2013 , the induced current of $500 \mathrm{~mA}$ was measured, as well as $50 \mathrm{~V}$ voltage at the frequency $f=621 \mathrm{kHz}$ due to $\mathrm{AM}$ antenna broadcasting at this frequency (Spanish national radio broadcast) from the nearby mountain [6]. When they lubricated the wires of steel cables supporting the spreader and thus isolated the spreader from crane structure by inserting some impedance, the problem was temporarily solved. The crane structure is presented in Figure 2, and the instrument measuring the induced voltage is presented in Figures 3 and 4 . The further work presented in this paper was initiated by coworking on this problem.

At the island Oahu (Hawaii), the crane operator experienced electrical shock while using it to lower steel peelings into the canal [2]. The crane had the boom of $33.5 \mathrm{~m}$ length

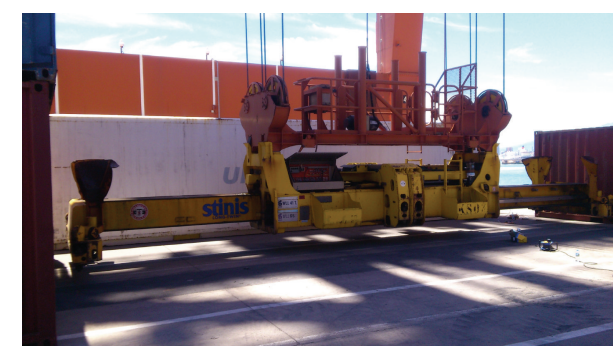

FIGURE 3: Measurement site.

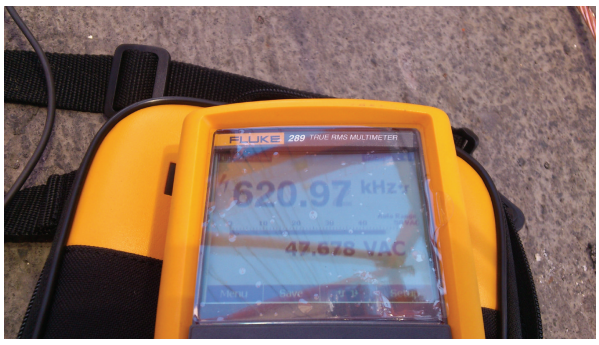

FIGURE 4: Instrument measuring induced voltage of about $50 \mathrm{~V}$ at about $621 \mathrm{kHz}$.

and was near transmitter broadcasting five AM and one FM channel at the frequencies from 30 to $100 \mathrm{MHz}$ with the total power $117 \mathrm{~kW}$ and antenna of height $h=122 \mathrm{~m}$.

In $50 \mathrm{t}$ rough-terrain crane in Japan [3] induced voltages in the hook up to $1.2 \mathrm{kV}$ and several amperes measured in the boom of this crane were noticed. It was positioned in the far field of the transmitter operating at frequency $f=624 \mathrm{kHz}$, at the distance where the measured electric field was $E_{0}=$ $0.79 \mathrm{~V} / \mathrm{m}$. The case is examined further in this paper. In [3] the solution to put acrylic plate under outrigger floats is given, and also another solution to add a plain knit grounding wire in the hook was suggested.

On the building site in Rio de Janeiro (Brazil) where two cranes were used, workers reported electrical shock and skin burns when they touched the boom of the large crawler crane, and electronic systems of the smaller truck-based crane became inoperative [7]. The grounding of the vehicle did not solve the problem. It was due to AM transmitters operating with the power of $100 \mathrm{~kW}$ at $f_{1}=1280 \mathrm{kHz}$ (station 1) and at $f_{2}=900 \mathrm{kHz}$ (station 2), approximately 230 meters from the building site. Practical solution to the problem was a coil wounded around the hydraulic jack of the crane, with one end connected to the hook and the other grounded to a nearby point.

EMI problem was noticed also by workers using a large crane during the assembly phase of the turbine component, while building wind power plant in Turkey [5], nearby AM transmitter operating at $f=702 \mathrm{kHz}$ with the power of $550 \mathrm{~kW}$. Steel tower of the turbine had 80 meters of height, and the rotor with three blades 44 meters long. The main boom of the crane was $110 \mathrm{~m}$ long and there was also a shorter auxiliary boom. Crane site was $8 \mathrm{~km}$ from the transmitter. Additional grounding was used to mitigate the induced effects. 


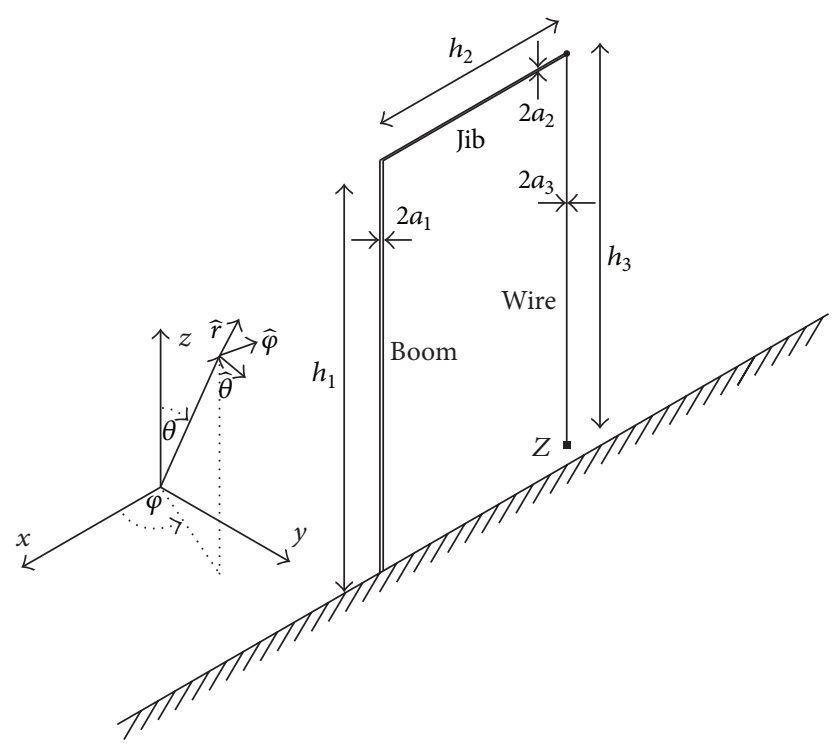

FIGURE 5: Simple wire antenna model of the crane in external electromagnetic field.

All the mentioned problems may be treated as receiving wire antenna in external electromagnetic field, so that some of the codes may be used as NEC [9], AWAS [10], and so forth. Programs AWAS and SPAN [11] are used in this paper to calculate induced currents and voltages for the given examples.

\section{Crane Acting as Receiving Antenna in External Electromagnetic Field}

To model the crane structure as wire antenna in external electromagnetic field, it is necessary to determine if the field is near or far. For all the mentioned cases, the construction work is done in the far field of transmitting antenna, at a few hundreds of meters or even kilometers away [12, 13]. Near field is in fact prohibited area with electric field values much over the occupational/controlled limits according to regulations. It should be noticed that mentioned problems occur in the area with electric field values below the public limits for these frequencies [14]. Let us assume that receiving antenna is in a plane wave electromagnetic field, so that electric field is

$$
\begin{aligned}
E_{s} & =E_{0} \\
& \cdot \exp [-j k(x \sin \theta \cos \varphi+y \sin \theta \sin \varphi+z \cos \theta)],
\end{aligned}
$$

where $\theta$ and $\varphi$ are spherical coordinates, $x, y$, and $z$ are Descartes coordinates given in Figure $5, E_{0}$ is the maximum electric field strength, $k=2 \pi / \lambda$ is the phase constant, $\lambda=c / f$ is the wavelength, and $c$ is the speed of light.

The crane is treated as wire antenna structure at perfectly conducting ground. To model a $50 \mathrm{t}$ rough-terrain crane [3] with cylindrical segments, the boom segment is taken of height $h_{1}=50.5 \mathrm{~m}$ and radius $a_{1}=37.5 \mathrm{~cm}$, the jib of length $h_{2}=26 \mathrm{~m}$ and radius $a_{2}=20 \mathrm{~cm}$, and the wire of length $h_{3}$
TABLE 1: Induced voltages in case A.

\begin{tabular}{lccc}
\hline \multicolumn{3}{c}{ Voltage $(\mathrm{V})$} \\
$f(\mathrm{kHz})$ & $\theta=0, \varphi=0$ & $\theta=\pi / 2, \varphi=0$ & $\theta=\pi / 2, \varphi=\pi / 2$ \\
\hline 500 & 25.74 & 48.61 & 41.20 \\
550 & 33.85 & 70.55 & 61.65 \\
600 & 47.77 & 110.66 & 99.10 \\
650 & 78.30 & 203.10 & 185.60 \\
700 & 196.40 & 574.84 & 533.95 \\
720 & 346.53 & 1067.93 & 997.05 \\
726 & 370.75 & 1159.83 & 1085.61 \\
727 & 370.34 & 1161.98 & 1087.43 \\
728 & 368.51 & 1159.19 & 1085.32 \\
735 & 325.62 & 1043.23 & 1069.28 \\
750 & 207.44 & 691.59 & 978.15 \\
800 & 76.63 & 294.23 & 650.84 \\
850 & 44.39 & 198.67 & 512.33 \\
900 & 29.95 & 158.59 & 421.28 \\
1000 & 16.15 & 125.72 & 279.76 \\
\hline
\end{tabular}

$=48 \mathrm{~m}$ and radius $a_{3}=1.5 \mathrm{~cm}$. This is the first examined crane, denoted by case A. For the second examined crane, denoted by case $B$, lengths are the same for all segments, but radii are $a_{1}=15 \mathrm{~cm}, a_{2}=10 \mathrm{~cm}$, and $a_{3}=2.5 \mathrm{~cm}$. The hook is at height $2.5 \mathrm{~m}$ above the ground, in both cases. If using FDTD method as in [3], calculated induced voltages are about $600 \mathrm{~V}$, whereas measured results from [3] are up to $1.2 \mathrm{kV}$, and induced currents are of several amperes, if the maximum electric field is $E_{0}=0.79 \mathrm{~V} / \mathrm{m}$ and $f=624 \mathrm{kHz}$. Results for this crane are calculated in this paper using AWAS [10], based on Pocklington's equation [15] and polynomial approximation of the currents [16]. The induced voltages are given in Table 1 for case A and in Table 2 for case B, for the range of frequencies of interest and the three propagation directions. These show that frequencies producing the largest voltages differ in the two cases, so as the maximum of induced voltages. At $f=727 \mathrm{kHz}$ maximum voltage $U_{\operatorname{maxA}}=1161.98 \mathrm{~V}$ is calculated in case $\mathrm{A}$, whereas at $f=676 \mathrm{kHz}$ voltage $U_{\operatorname{maxB}}$ $=1433.24 \mathrm{~V}$ in case $\mathrm{B}$, both for the propagation directions $\theta=\pi / 2, \varphi=0$.

The induced currents along the structure are given in Figures 6-11, starting from the base of the boom, along the jib, and down the wire to the hook at the end. These results are given in Figure 6 for the electric field $E_{\theta}=0.79 \mathrm{~V} / \mathrm{m}, E_{\varphi}=0$, for the propagation directions $\theta=0, \varphi=0$, in Figure 7 for $\theta=\pi / 2, \varphi=0$, and in Figure 8 for $\theta=\pi / 2, \varphi=\pi / 2$, for case A (total length along the crane in that case is $124.5 \mathrm{~m}$ ). For $E_{\theta} \neq 0, E_{\varphi}=0, \theta=0, \varphi=\pi / 2$, there are no voltages or currents induced, but this is not the case in real situation. In fact $\theta$ is near $\pi / 2$ and any $\varphi$ may exist during crane operation, as both antenna structures are vertically positioned at the ground.

It is shown that at some frequencies the phenomenon will not be noticed at all. The maximum of induced current at the base of the boom is about 5 amperes, and it occurs for the frequency $f=727 \mathrm{kHz}$ and the propagation directions $\theta=$ 
TABLE 2: Induced voltages in case B.

\begin{tabular}{lccc}
\hline \multicolumn{3}{c}{ Voltage $(\mathrm{V})$} \\
$f(\mathrm{kHz})$ & $\theta=0, \varphi=0$ & $\theta=\pi / 2, \varphi=0$ & $\theta=\pi / 2, \varphi=\pi / 2$ \\
\hline 500 & 28.59 & 58.06 & 50.83 \\
550 & 40.80 & 91.95 & 82.68 \\
600 & 68.07 & 171.31 & 157.33 \\
640 & 139.45 & 385.12 & 358.90 \\
670 & 420.36 & 1248.21 & 1174.41 \\
675 & 474.24 & 1426.01 & 1343.43 \\
676 & 475.32 & 1433.24 & 1350.07 \\
677 & 472.50 & 1427.73 & 1345.88 \\
680 & 443.51 & 1350.95 & 1274.10 \\
700 & 194.54 & 623.25 & 590.86 \\
750 & 67.36 & 246.68 & 236.49 \\
800 & 39.12 & 165.53 & 160.24 \\
850 & 26.58 & 131.73 & 128.43 \\
900 & 19.34 & 114.05 & 111.79 \\
1000 & 11.03 & 97.61 & 96.55 \\
\hline
\end{tabular}

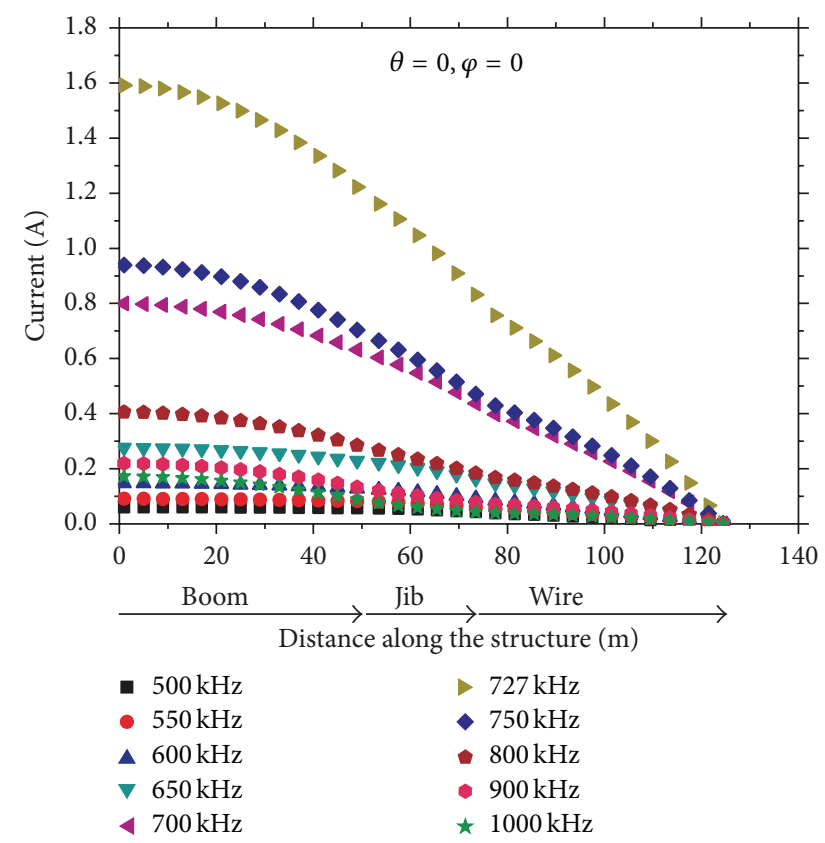

FIGURE 6: Induced currents versus length from the boom base, case A, directions $\theta=0, \varphi=0$.

$\pi / 2, \varphi=0$, in case A. This current is about 5.7 amperes for $f=676 \mathrm{kHz}$ in case B. It should be noticed that for $\pm 30 \mathrm{kHz}$ currents along the structure are about one-half of the value at $f=727 \mathrm{kHz}$, as shown in Figure 7 .

There is a difference in current results at segments beginnings/ends, if compared to FDTD results [3]. There are no discontinuities in currents results due to Kirchhoff's law for currents at nodes which is satisfied in the applied procedure. The chosen polynomial degree is $n=3$. In case $\mathrm{B}$, for $\pm 30 \mathrm{kHz}$, currents along the structure are about one-third of the value at $f=676 \mathrm{kHz}$, as shown in Figure 10. Resonant

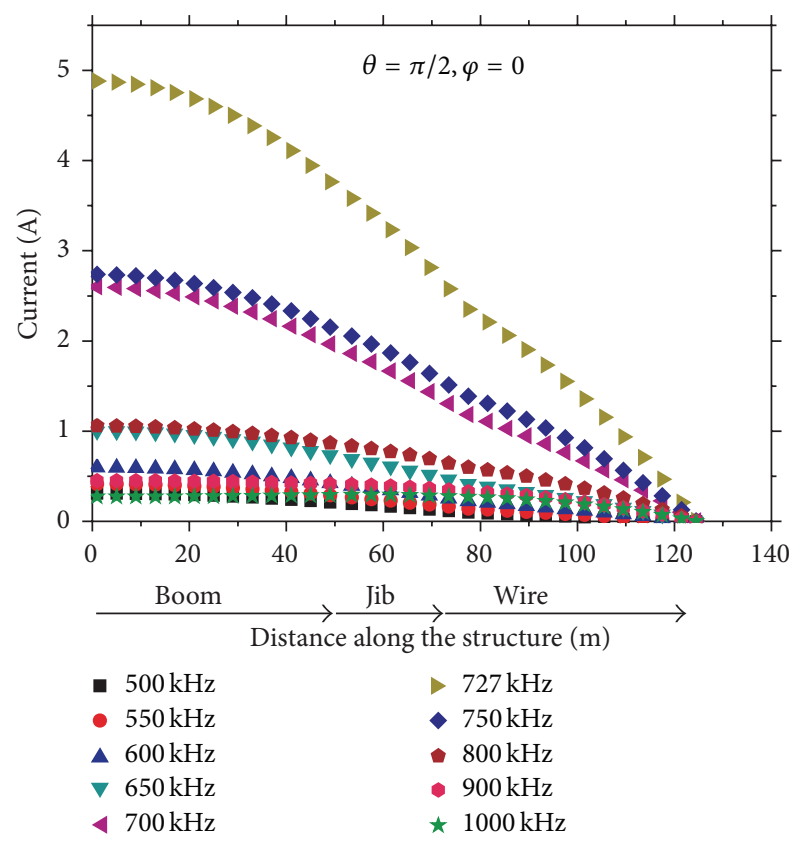

FIGURE 7: Induced currents versus length from the boom base, case A, directions $\theta=\pi / 2, \varphi=0$.

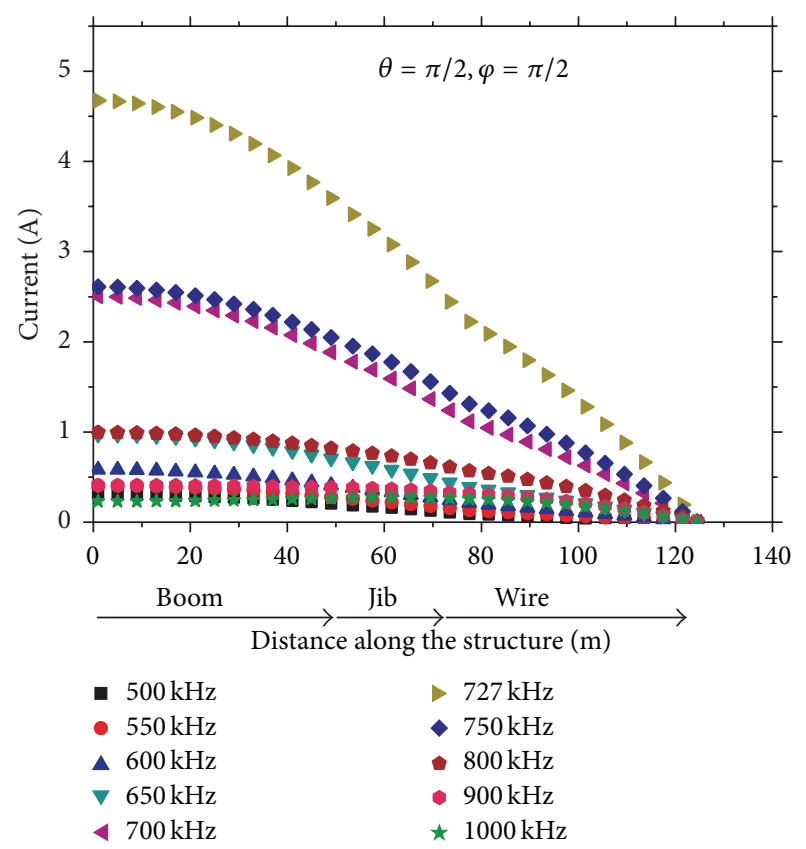

FIgURE 8: Induced currents versus length from the boom base, case A, directions $\theta=\pi / 2, \varphi=\pi / 2$.

characteristics of this structure may be noticed also in results for the induced voltages versus frequency in Figures 12 and 13, for the two cases A and B.

From the calculation of induced currents and voltages for these two cases it may be noticed that the change in segments cross section dimensions has great influence. Change of wire diameter, determined also with mechanical constraints, changes resonant frequency and in this way the problem 


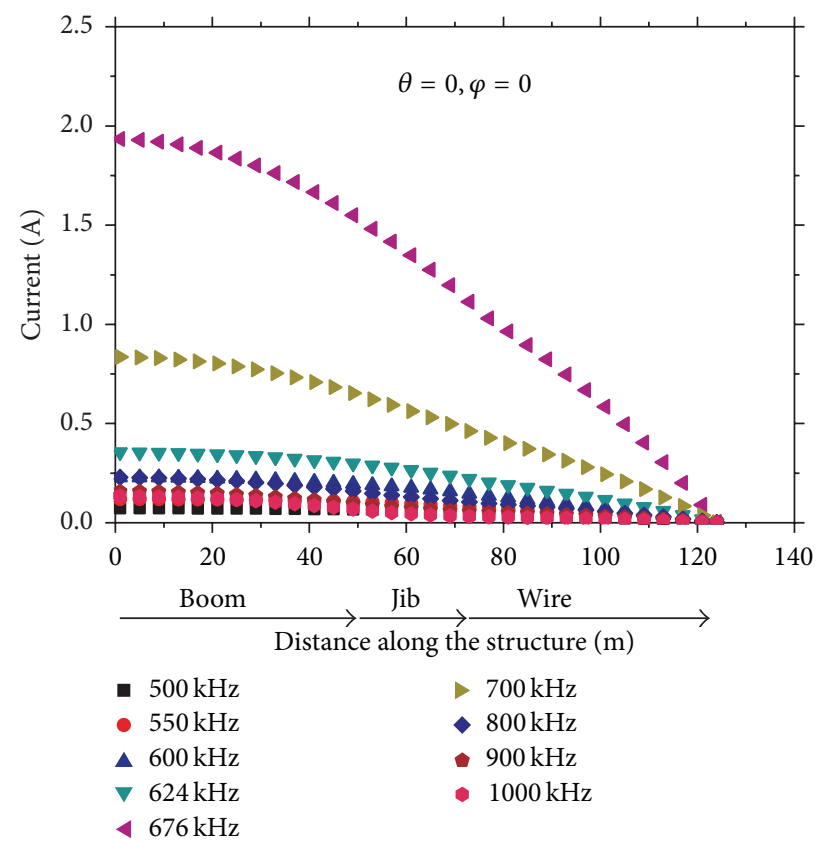

Figure 9: Induced currents versus length from the boom base, case $\mathrm{B}$, directions $\theta=0, \varphi=0$.

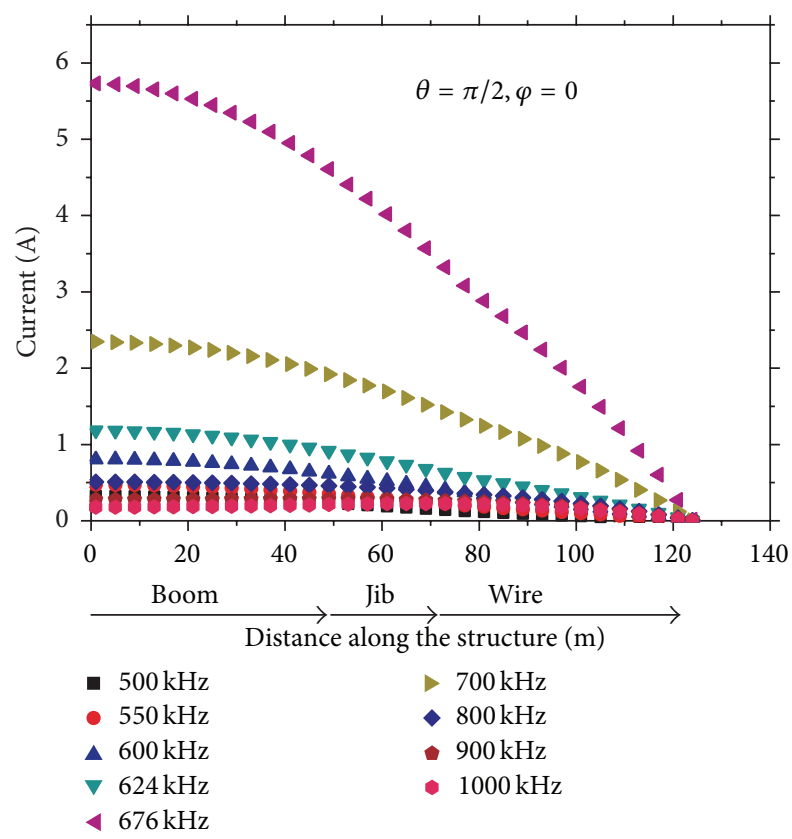

FIGURE 10: Induced currents versus length from the boom base, case $\mathrm{B}$, directions $\theta=\pi / 2, \varphi=0$.

might be mitigated. Another approach may be an adequate choice of crane configuration after the structure is analyzed and induced voltages and currents are computed.

\section{Computation Procedure Based on Hallen's Equation}

Magnetic vector-potential of a wire antenna structure with $n$ linear cylindrical segments, having current distribution

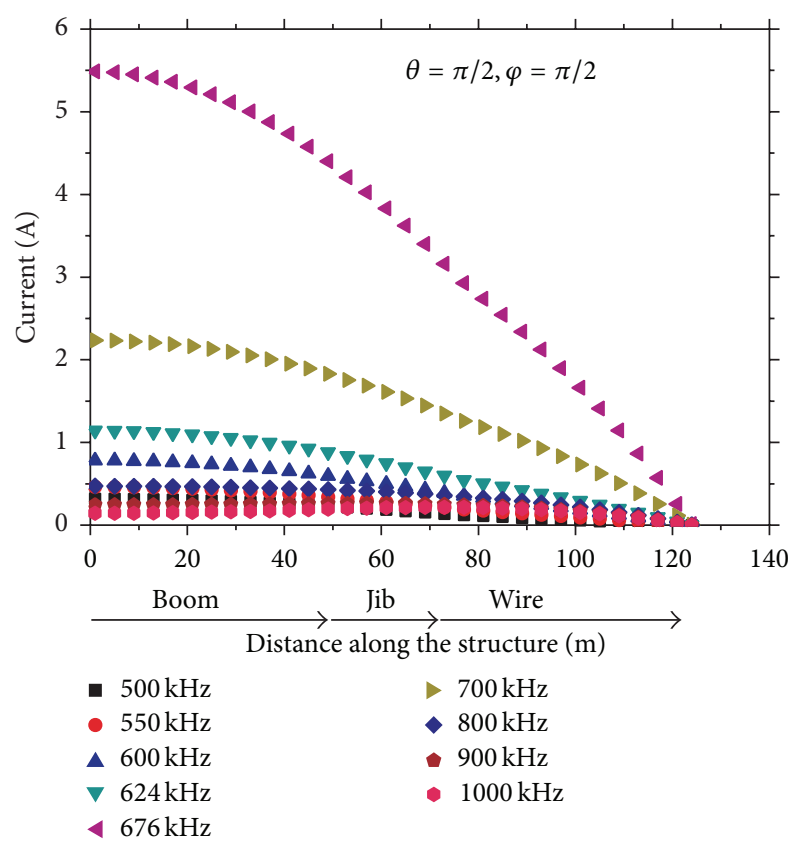

FIGURE 11: Induced currents versus length from the boom base, case B, directions $\theta=\pi / 2, \varphi=\pi / 2$.

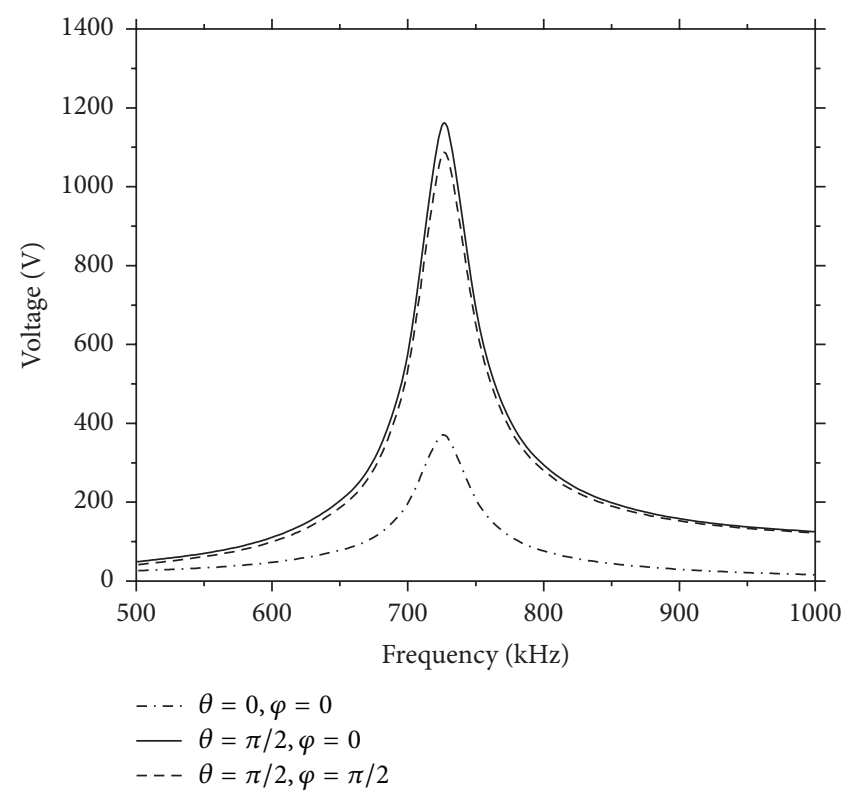

FIGURE 12: Open-circuit voltage in case A for $E_{\theta}=0.79 \mathrm{~V} / \mathrm{m}, E_{\varphi}=0$ and propagation directions: $\theta=0$ and $\varphi=0 ; \theta=\pi / 2$ and $\varphi=0$; $\theta=\pi / 2$ and $\varphi=\pi / 2$.

$I_{z_{m}}\left(z_{m}^{\prime}\right)$ along $m$ th segment, is obtained as the sum of $n$ components. Each component $A\left(z_{m}\right)$ is in the direction of $m$ th segment, having length $h_{m}$ and radius $a_{m}$, so that

$$
A\left(z_{m}\right)=\frac{\mu}{4 \pi} \int_{0}^{h_{m}} I_{z_{m}}\left(z_{m}^{\prime}\right) \frac{\exp \left(-j k r_{m, m}\right)}{r_{m, m}} \mathrm{~d} z_{m}^{\prime},
$$




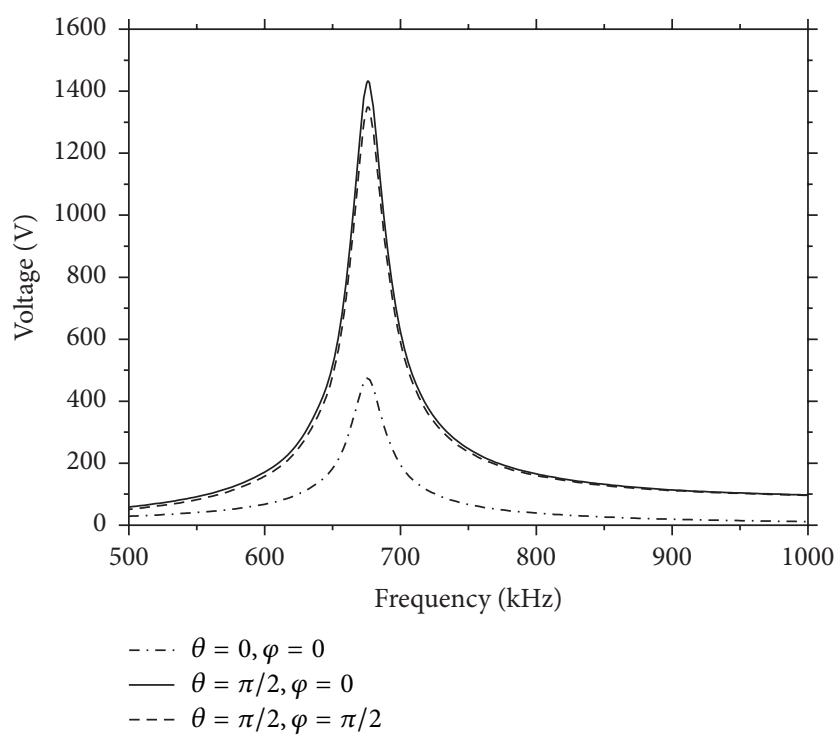

FIGURE 13: Open-circuit voltage in case B for $E_{\theta}=0.79 \mathrm{~V} / \mathrm{m}, E_{\varphi}=0$ and propagation directions: $\theta=0$ and $\varphi=0 ; \theta=\pi / 2$ and $\varphi=0$; $\theta=\pi / 2$ and $\varphi=\pi / 2$.

where $r_{m, m}=\left[x_{m}{ }^{2}+y_{m}{ }^{2}+\left(z_{m}-z_{m}^{\prime}\right)^{2}\right]^{1 / 2}$ is the distance between the point $M\left(x_{m}, y_{m}, z_{m}\right)$ and the point $P\left(0,0, z_{m}^{\prime}\right)$ along the axis of the $m$ th segment, for $m=1, \ldots, n$.

The total magnetic vector-potential is

$$
A=\sum_{m=1}^{n} A\left(z_{m}\right) .
$$

Descartes components of magnetic vector-potential originating from the $l$ th segment current are recalculated into the $m$ th segment Descartes coordinate system as

$$
\begin{aligned}
& A_{x_{m}}=\sum_{l, l \neq m} \sin \psi_{l, m} \sin \theta_{l, m} A\left(z_{l}\right), \\
& A_{y_{m}}=-\sum_{l, l \neq m} \cos \psi_{l, m} \sin \theta_{l, m} A\left(z_{l}\right), \\
& A_{z_{m}}=A\left(z_{m}\right)+\sum_{l, l \neq m} \cos \theta_{l, m} A\left(z_{l}\right),
\end{aligned}
$$

where $\theta_{l, m}, \psi_{l, m}$, and $\varphi_{l, m}$ are Euler's angles between the $l$ th and $m$ th segments of Descartes coordinate systems. Boundary condition for the tangential component of electric field at the conductive surface and Lorenz gauge condition are used, so that

$$
\begin{aligned}
& A_{z_{m}}=C_{1 m} \cos \left(k z_{m}\right)+C_{2 m} \sin \left(k z_{m}\right)-F\left(z_{m}\right), \\
& F\left(z_{m}\right) \\
& =\left.\int_{0}^{z_{m}}\left(\frac{\partial A_{x_{m}}}{\partial x_{m}}+\frac{\partial A_{y_{m}}}{\partial y_{m}}\right)\right|_{\substack{x_{m}=a_{m} \\
y_{m}=0 \\
z_{m}=s}} \cos \left[k\left(z_{m}-s\right)\right] \mathrm{d} s
\end{aligned}
$$

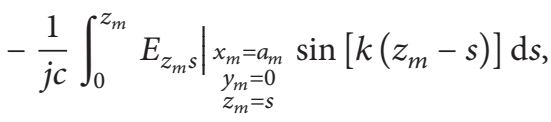

where $C_{1 m}$ and $C_{2 m}$ are complex constants and $E_{z_{m} s}$ is the external electric field component at the surface of the $m$ th segment. Function (6) is written as

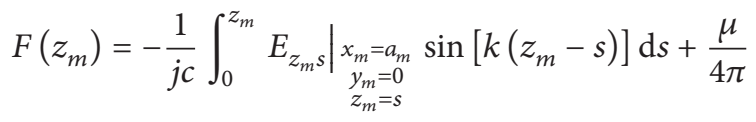

$$
\begin{aligned}
& \cdot \int_{0}^{h_{l}} I_{z_{l}}\left(z_{l}^{\prime}\right) \int_{0}^{z_{m}} \cos \left[k\left(z_{m}-s\right)\right] \\
& \cdot\left(\frac{\partial}{\partial z_{l}^{\prime}}+\cos \theta_{l, m} \frac{\partial}{\partial z_{m}}\right) \\
& \left.\frac{\exp \left(-j k r_{l, m}\right)}{r_{l, m}}\right|_{\substack{x_{m}=a_{m} \\
y_{m}=0 \\
z_{m}=s}} \mathrm{~d} s \mathrm{~d} z_{l}^{\prime}
\end{aligned}
$$

which results in the system of integrodifferential equations of Hallen's type [17] for the currents along this structure:

$$
\begin{gathered}
\sum_{l=1}^{n} \int_{0}^{h_{l}} I_{z_{l}}\left(z_{l}^{\prime}\right) K_{m, l}\left(z_{m}, z_{l}^{\prime}\right) \mathrm{d} z_{l}^{\prime}-\frac{4 \pi C_{1 m}}{\mu} \cos \left(k z_{m}\right) \\
-\frac{4 \pi C_{2 m}}{\mu} \sin \left(k z_{m}\right) \\
=\left.\frac{4 \pi}{j c \mu} \int_{0}^{z_{m}} E_{z_{m} s}\right|_{\substack{x_{m}=a_{m} \\
y_{m}=0 \\
z_{m}=s}} \sin \left[k\left(z_{m}-s\right)\right] \mathrm{d} s \\
-\frac{4 \pi}{j c \mu} \sum_{i=1}^{n_{Z}} Z^{(i)} I\left(z_{m}{ }^{(i)}\right) \sin \left[k\left(z_{m}-z_{m}{ }^{(i)}\right)\right]
\end{gathered}
$$

for $m=1, \ldots, n$, whereas integral kernels are for $l \neq m$

$$
\begin{gathered}
K_{m, l}\left(z_{m}, z_{l}^{\prime}\right)=\left.\cos \theta_{l, m} \frac{\exp \left(-j k r_{l, m}\right)}{r_{l, m}}\right|_{\substack{x_{m}=a_{m} \\
y_{m}=0}} \int_{0}^{z_{m}} \cos \left[k\left(z_{m}-s\right)\right]\left[\frac{\partial}{\partial z_{l}^{\prime}}+\cos \theta_{l, m} \frac{\partial}{\partial z_{m}}\right] \\
.\left.\frac{\exp \left(-j k r_{l, m}\right)}{r_{l, m}}\right|_{\substack{x_{m}=a_{m} \\
y_{m}=0 \\
z_{m}=s}} \mathrm{~d} s,
\end{gathered}
$$

and for $l=m$

$$
K_{l, l}\left(z_{l}, z_{l}^{\prime}\right)=\left.\frac{\exp \left(-j k r_{l, l}\right)}{r_{l, l}}\right|_{\substack{x_{l}=a_{l} \\ y_{l}=0}}
$$

In (8), $Z^{(i)}$ is the concentrated impedance at point $z_{m}{ }^{(i)}$ along the $i$ th segment, for $i=1, \ldots, n_{z}$, where $n_{z}$ is the total number of concentrated impedances for that segment. This system of equations is solved by Point matching method as the Method of Moments (MoM) [18] and matching points are equidistantly positioned at the surface of each segment, including the beginnings and ends of segments. Currents are assumed to be distributed along the axes of segments, although they are flowing along the conductive surface of 
the structure, in order to avoid singularities in integrals. Distributions of the currents are unknowns and they are approximated by polynomials [16].

The distance between the matching point $M\left(x_{m}, y_{m}, z_{m}\right)$ at $m$ th segment surface and the point $P_{l}\left(0,0, z_{l}^{\prime}\right)$ along the axis of $l$ th segment, where the elementary current source is positioned, for $l \neq m$ is

$$
\begin{aligned}
& r_{l, m}=\left[\left(x_{m}-x_{b l, m}-z_{l}^{\prime} \sin \psi_{l, m} \sin \theta_{l, m}\right)^{2}\right. \\
& +\left(y_{m}-y_{b l, m}-z_{l}^{\prime} \cos \psi_{l, m} \sin \theta_{l, m}\right)^{2} \\
& \left.+\left(z_{m}-z_{b l, m}-z_{l}^{\prime} \cos \theta_{l, m}\right)^{2}\right]^{1 / 2}
\end{aligned}
$$

and for $l=m$

$$
r_{l, l}=\left[x_{l}^{2}+y_{l}^{2}+\left(z_{l}-z_{l}^{\prime}\right)^{2}\right]^{1 / 2}
$$

$P_{b}\left(x_{b l, m}, y_{b l, m}, z_{b l, m}\right)$ is the beginning of the lth segment, that is, its coordinate system origin, recalculated into the $m$ th segment coordinate system. Kirchhoff's current law is satisfied for all the nodes. For $n_{D}$ antenna segments incident to node $D$, Kirchhoff's law is

$$
\sum_{r=1}^{n_{D}} \sum_{s=1}^{n_{r}} B_{r s}\left(\frac{z_{r}}{h_{r}}\right)^{s}=0,
$$

for unknown complex coefficients $B_{r s}$ and chosen polynomial degree $n_{r}$ in the approximation of current along the $r$ th segment. Coordinate of the beginning/end of $r$ th segment is $z_{r}$ and $h_{r}$ is the segment length. Electric potential is calculated from the following expression:

$$
\begin{aligned}
& V_{z_{m}}=-j c\left[C_{1 m} \sin \left(k z_{m}\right)-C_{2 m} \cos \left(k z_{m}\right)\right] \\
& +\left.\int_{0}^{z_{m}} E_{z_{m} s}\right|_{\substack{x_{m}=a_{m} \\
y_{m}=0 \\
z_{m}=s}} \cos \left[k\left(z_{m}-s\right)\right] \mathrm{d} s \\
& +\left.j c \int_{0}^{z_{m}}\left(\frac{\partial A_{x_{m}}}{\partial x_{m}}+\frac{\partial A_{y_{m}}}{\partial y_{m}}\right)\right|_{\substack{x_{m}=a_{m} \\
y_{m}=0 \\
z_{m}=s}} \\
& \cdot \sin \left[k\left(z_{m}-s\right)\right] \mathrm{d} s-\sum_{i=1}^{n_{Z}} Z^{(i)} I\left(z_{m}{ }^{(i)}\right) \\
& \cdot \cos \left[k\left(z_{m}-s\right)\right] \text {. }
\end{aligned}
$$

\section{Tower Crane and Turn-Over Crane Examples}

More complex crane structures are modelled and system of (8) is solved for each example using the presented procedure. Some interesting results for tower crane and turn-over crane [1] are obtained. For the crane in Figure 14 in far electromagnetic field, $E_{\theta}=1 \mathrm{~V} / \mathrm{m}, E_{\varphi}=0$, for $f=1 \mathrm{MHz}$ and different propagation directions, effective current along main tower segment of the height $l=34 \mathrm{~m}$ is presented in Figure 15, for

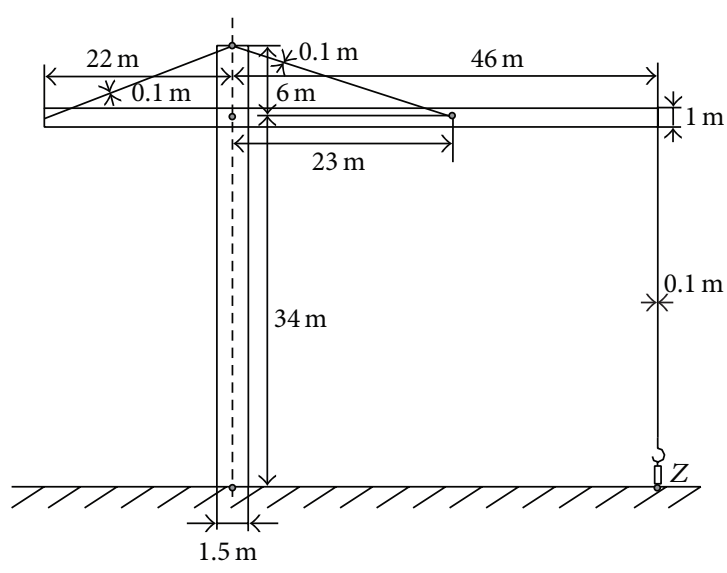

Figure 14: Tower crane model.

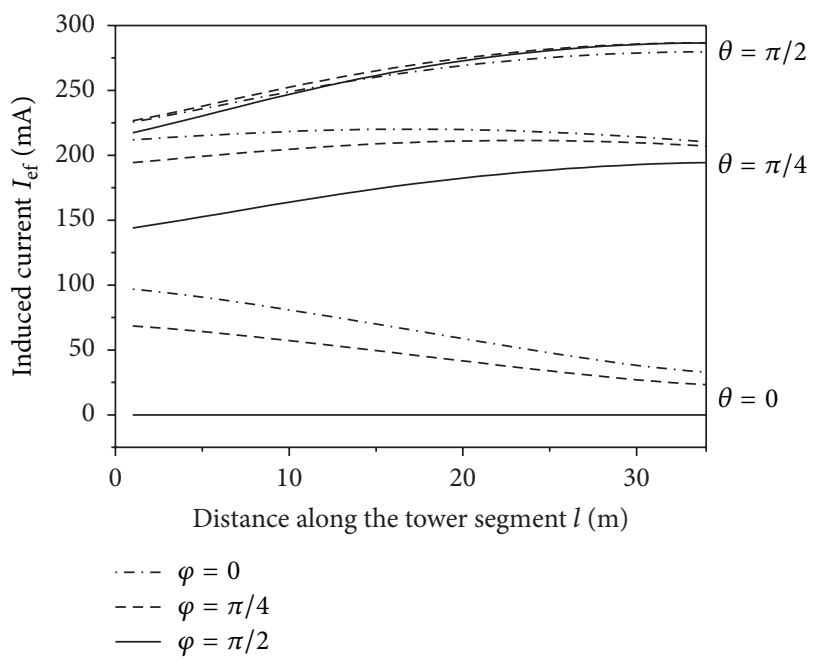

FIGURE 15: Induced current along the main tower segment of the tower crane.

the polynomial degree $n=5$ and impedance $Z=100 \Omega$. If the turn-over crane given in Figure 16 is in the same field, the effective current along boom segment of the height $l=32 \mathrm{~m}$ is presented in Figure 17.

For $f=1 \mathrm{MHz}$ the wavelength is $\lambda=300 \mathrm{~m}$. The results for different frequencies are analyzed and it is obtained that for the tower crane example the maximum currents along the structure and maximum touch voltages at the hook are at $f=725.8 \mathrm{kHz}$ and for the turn-over crane are at $f=1.49 \mathrm{MHz}$. Eight segments and seven nodes were chosen to model the tower crane at perfectly conducting ground, whereas six segments and six nodes were chosen for the turnover crane.

\section{Reloading Bridge and Portal-Harbor Crane Examples}

It is important to consider reloading bridges, portal-harbor cranes, and container cranes, as they may be permanently in the field of MW transmitter a few kilometers from the crane's 


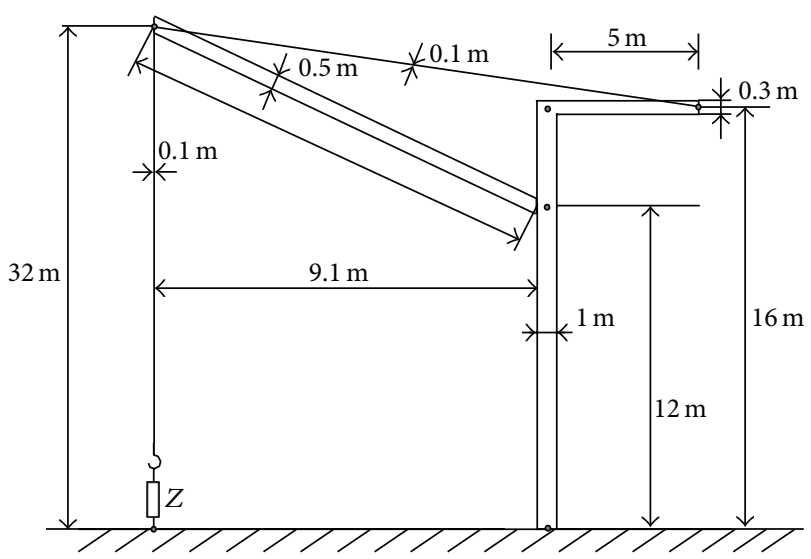

FIgURE 16: Turn-over crane model.

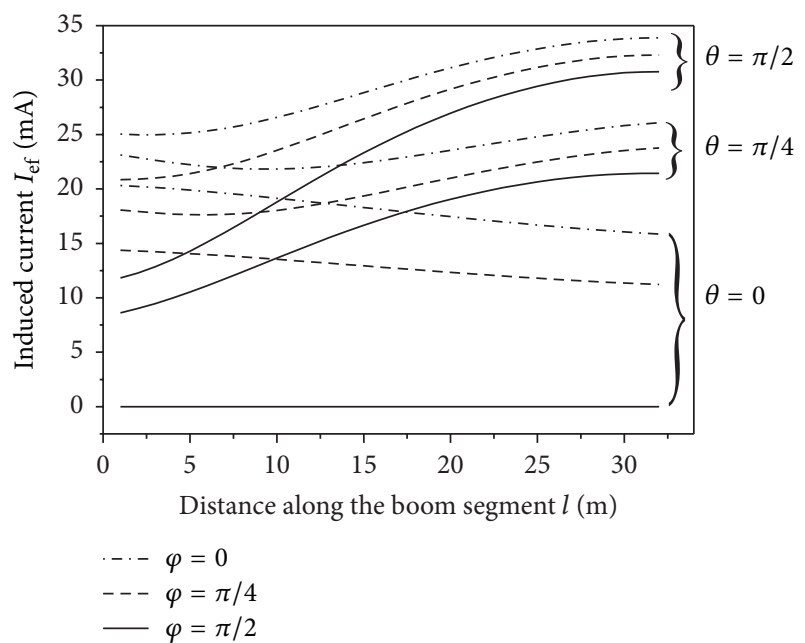

FIGURE 17: Induced current along the boom segment of the turnover crane.

site. Electric fields in such cases are often of the order of V/m, depending on the transmitter power and vicinity. Positions of such cranes should be carefully selected based on modeling of the specific problem. Induced effects may pose great risk in some cases $[19,20]$.

An example of the reloading bridge with its dimensions is given in Figure 18. Portal-harbor crane of dimensions very similar to the described rail-mounted container crane is presented in Figure 19. For these cranes positioned in the far field, for $E_{\theta}=1 \mathrm{~V} / \mathrm{m}, E_{\varphi}=0, \theta=\pi / 2$, and $\varphi=$ $\pi / 2$, touch voltages and induced currents are calculated for the polynomial degree $n=5$ and impedance $Z=1000 \Omega$. Results are presented in Figures 20 and 21. Induced voltage for the portal-harbor crane is about $50 \mathrm{~V}$ for frequency $f=$ $621 \mathrm{kHz}$, as measured in Tenerife (Spain), although this is just a simplified model.

The impedance which models a human touching the hook may be chosen according to the frequency of interest and using results from [21-25].

It may be concluded that the reloading bridge is more suitable, as it has less resonant frequencies, smaller values of

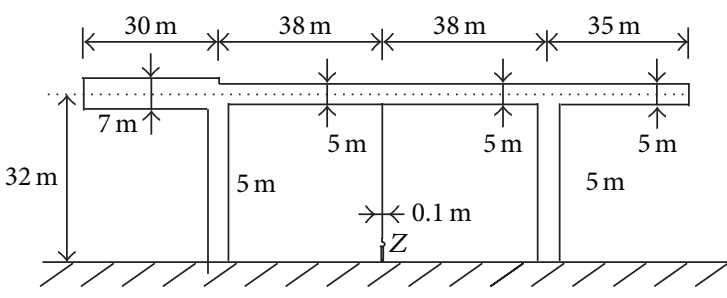

FIgURE 18: Reloading bridge model.

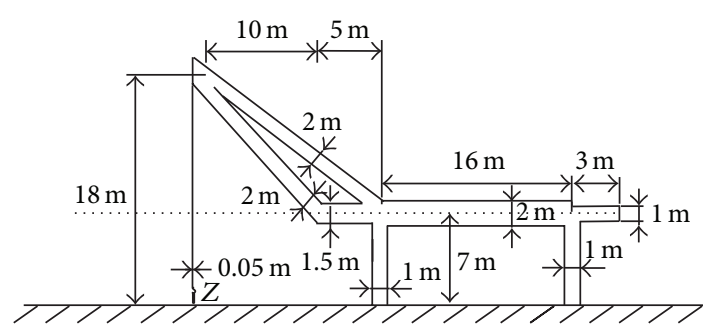

FIGURE 19: Portal-harbor crane model.

induced currents, and smaller values of touch voltages except in the narrow bandwidth around resonant frequency.

\section{Conclusion}

Currents up to several amperes and touch voltages up to about $1 \mathrm{kV}$ may be induced in large cranes nearby MW transmitters. At such frequencies these values may result in electrical shock, painful burns, and dangerous involuntary movements of workers, but also in faults in the automation and control systems of cranes. Analysis of such problems is presented in this paper as well as results for induced current and voltages for different configurations of crane structures.

Current distribution along the crane structure is determined using wire antenna model of the crane and electromagnetic theory approach. Currents along segments are approximated by polynomials. System of integral equations for the currents is solved using Point matching method. Results are in agreement with measured currents and voltages.

This approach may be used to analyze specific problems, especially before positioning of reloading bridges and container cranes nearby MW transmitters. Some practical solutions to this problem are also given in the paper.

\section{Conflict of Interests}

The author declares that there is no conflict of interests regarding the publication of this paper.

\section{Acknowledgment}

This paper is in the frame of research within Projects HUMANISM III 44004 and TR33008 (2011-2015) financed 


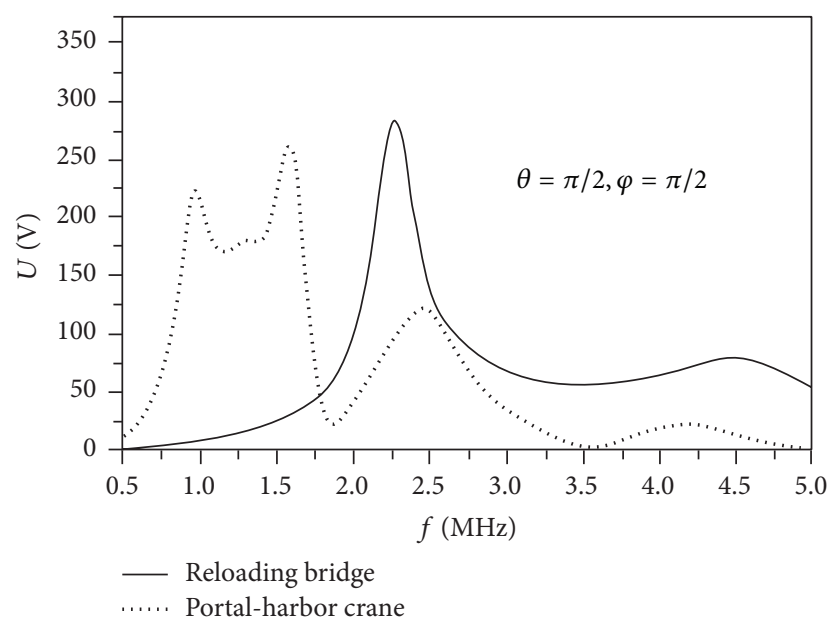

FIGURE 20: Induced voltage for reloading bridge and portal-harbor crane versus $f$.

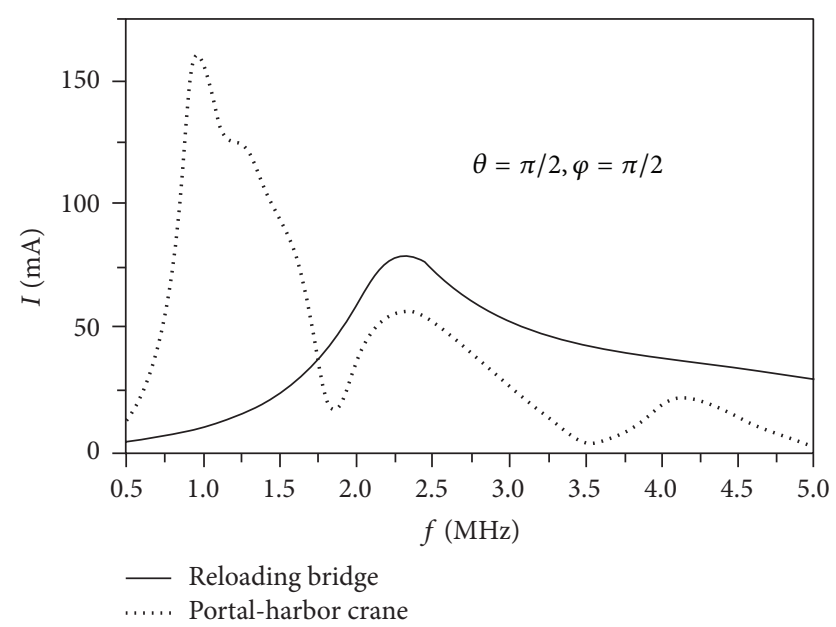

FIGURE 21: Induced current for reloading bridge and portal-harbor crane versus $f$.

by Ministry of Education, Science and Technological Development of the Republic of Serbia.

\section{References}

[1] V. Javor, "The calculation and elimination of undesirable electromagnetic field influence on cranes," in Proceedings of the 4th International Conference on Telecommunications in Modern Satellite, Cable and Broadcasting Services (TELSIKS '99), pp. 628-631, Nis, Serbia, October 1999.

[2] T. Denton, "Radiofrequency energy poses unseen hazard," Occupational Hazards, vol. 64, no. 12, pp. 45-47, 2002.

[3] T. Hajime, "Electromagnetic disturbance of large-scale crane due to medium wave broadcasting and countermeasures," Research Reports of National Institute of Industrial Safety, 2003.

[4] R. G. Olsen, J. B. Schneider, and R. A. Tell, "Radio frequency burns in the power system workplace," IEEE Transactions on Power Delivery, vol. 26, no. 1, pp. 352-359, 2011.

[5] F. Ustuner, "Practical papers, articles and application notes: interaction of an AM broadcast transmitter with a large crane posing health hazards: a real-world event analysis," IEEE Electromagnetic Compatibility Magazine, vol. 1, no. 2, pp. 41-49, 2012.

[6] V. Javor and M. Saranac, "Electromagnetic disturbances in conductive structures nearby transmitting antennas," in Proceedings of the 21st International Conference on Software, Telecommunications and Computer Networks (SoftCOM '13), pp. 1-5, IEEE, Primošten, Croatia, September 2013.

[7] M. B. Perotoni and R. M. Barreto, Resolving Safety-Critical EMI Problems between AM Transmitters and Cranes Using a 3D Field Solver, High Frequency Electronics, 2014.

[8] https://en.wikipedia.org/wiki/List_of_European_medium wave_transmitters.

[9] G. J. Burke and A. J. Poggio, "Numerical electromagnetics code (NEC)-method of moments," Tech. Rep. 116, Naval Ocean Systems Center, San Diego, Calif, USA, 1981.

[10] A. R. Djordjevic, M. B. Bazdar, T. K. Sarkar, and R. F. Harrington, AWAS for Windows: Analysis of Wire Antennas and Scatterers, Software and User's Manual, Artech House Books, Boston, Mass, USA, 1995.

[11] V. Javor and D. Velickovic, "Computer package for analysis of lightning electromagnetic field distribution for cage conductor structures," in Proceedings of the 26th International Conference on Lightning Protection (ICLP '02), Proceedings of Papers, pp. 382-387, Cracow, Poland, September 2002.

[12] O. P. Gandhi and I. Chatterjee, "Radio-frequency hazards in the VLF to MF band," Proceedings of the IEEE, vol. 70, no. 12, pp. 1462-1464, 1982

[13] W. Congjiang, L. Deming, W. Shuquan, W. Baosheng, and H. Baoge, "Environmental impact of electromagnetic radiation from the 10kW medium wave transmitter of Weihai Broadcasting Station," Journal of Environmental Sciences, vol. 7, no. 4, pp. 461-467, 1995.

[14] IEEE C.95.1-2005, IEEE Standard for safety levels with respect to human exposure to radio frequency electromagnetic fields, $3 \mathrm{kHz}$ to $300 \mathrm{GHz}$, IEEE, 2005.

[15] H. C. Pocklington, "Electrical oscillations in wires," Proceedings of the Cambridge Philosophical Society, Mathematical and Physical Sciences, vol. 9, pp. 324-332, 1897.

[16] B. D. Popovic, "Polynomial approximation of current along thin symmetrical cylindrical dipoles," Proceedings of the Institution of Electrical Engineers, vol. 117, no. 5, pp. 873-878, 1970.

[17] E. Hallén, "Theoretical investigations into the transmitting and receiving qualities of antennas," Nova Acta Regiae Societatis Scientiarum Upsaliensis Ser. IV, vol. 11, no. 4, pp. 1-44, 1938.

[18] R. F. Harrington, Field Computation by Moment Methods, section 6.2, Macmillan, New York, NY, USA, 1968.

[19] I. R. Bradby, Practical Experience in Radio Frequency Induced Ignition Risk Assessment for COMAH/DSEAR Compliance, Symposium Series no. 154, ABB Engineering Services, 2008.

[20] X.-T. Huang, "Study on the influence of induced high-voltage surge to tower crane by Baise MW broadcasting station and its protection," Journal of Safety Science and Technology, no. 2, pp. 146-151, 2010.

[21] H. Kanai, I. Chatterjee, and O. P. Gandhi, "Human body impedance for electromagnetic hazard analysis in the VLF to MF band," IEEE Transactions on Microwave Theory and Techniques, vol. 32, no. 8, pp. 763-772, 1984.

[22] D. A. Hill and J. A. Walsh, "Radio-frequency current through the feet of a grounded human," IEEE Transactions on Electromagnetic Compatibility, vol. 27, no. 1, pp. 18-23, 1985. 
[23] I. Chatterjee, D. Wu, and O. P. Gandhi, "Human body impedance and threshold currents for perception and pain for contact hazard analysis in the VLF-MF band," IEEE Transactions on Biomedical Engineering, vol. 33, no. 5, pp. 486-494, 1986.

[24] Y. Kamimura, K. Komori, M. Shoji, Y. Yamada, S. Watanabe, and Y. Yamanaka, "Human body impedance for contact current measurement in Japan," IEICE Transactions on Communications, vol. 88, no. 8, pp. 3263-3267, 2005.

[25] V. De Santis, P. A. Beeckman, D. A. Lampasi, and M. Feliziani, "Assessment of human body impedance for safety requirements against contact currents for frequencies up to $110 \mathrm{MHz}$," IEEE Transactions on Biomedical Engineering, vol. 58, no. 2, pp. 390396, 2011. 

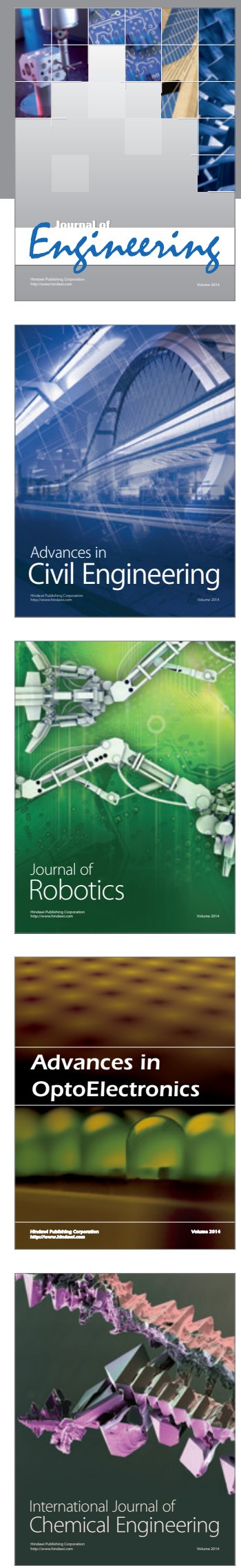

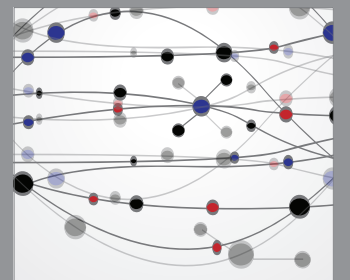

The Scientific World Journal
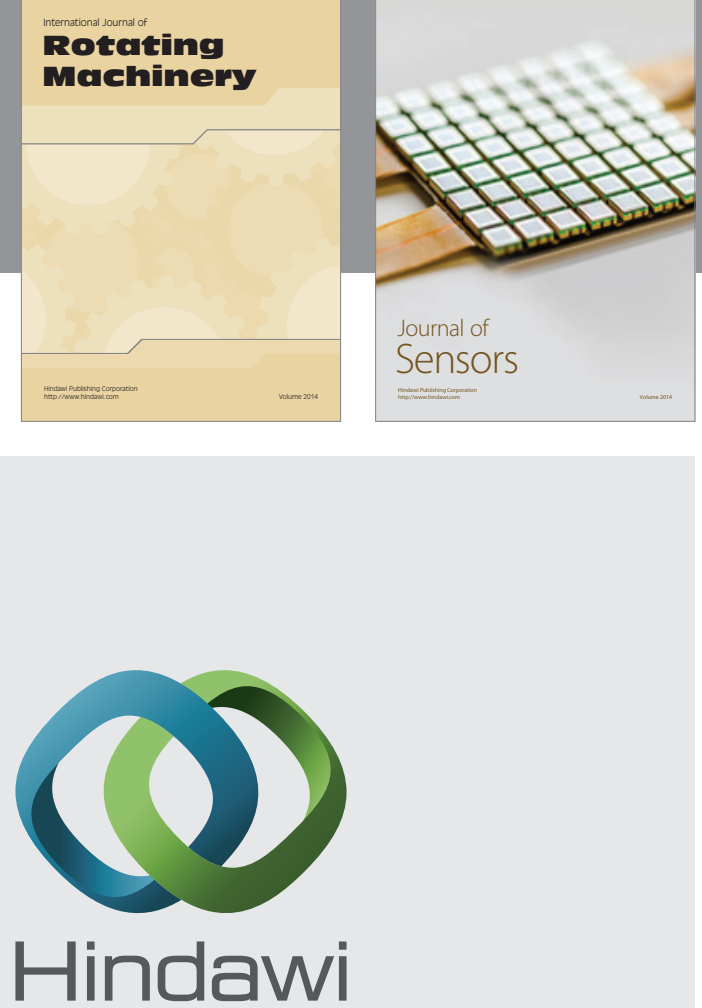

Submit your manuscripts at http://www.hindawi.com
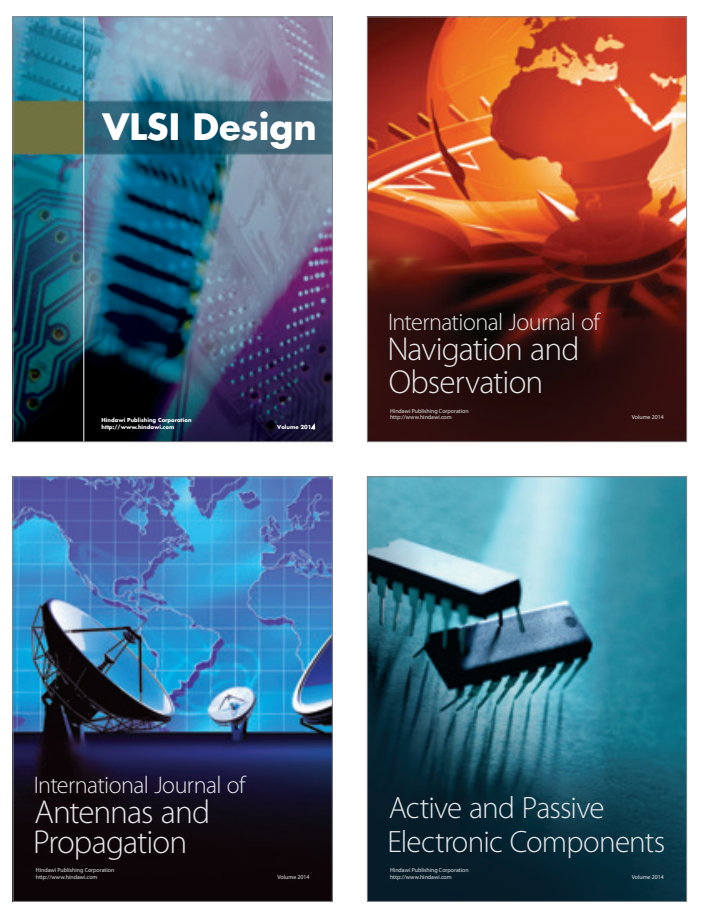
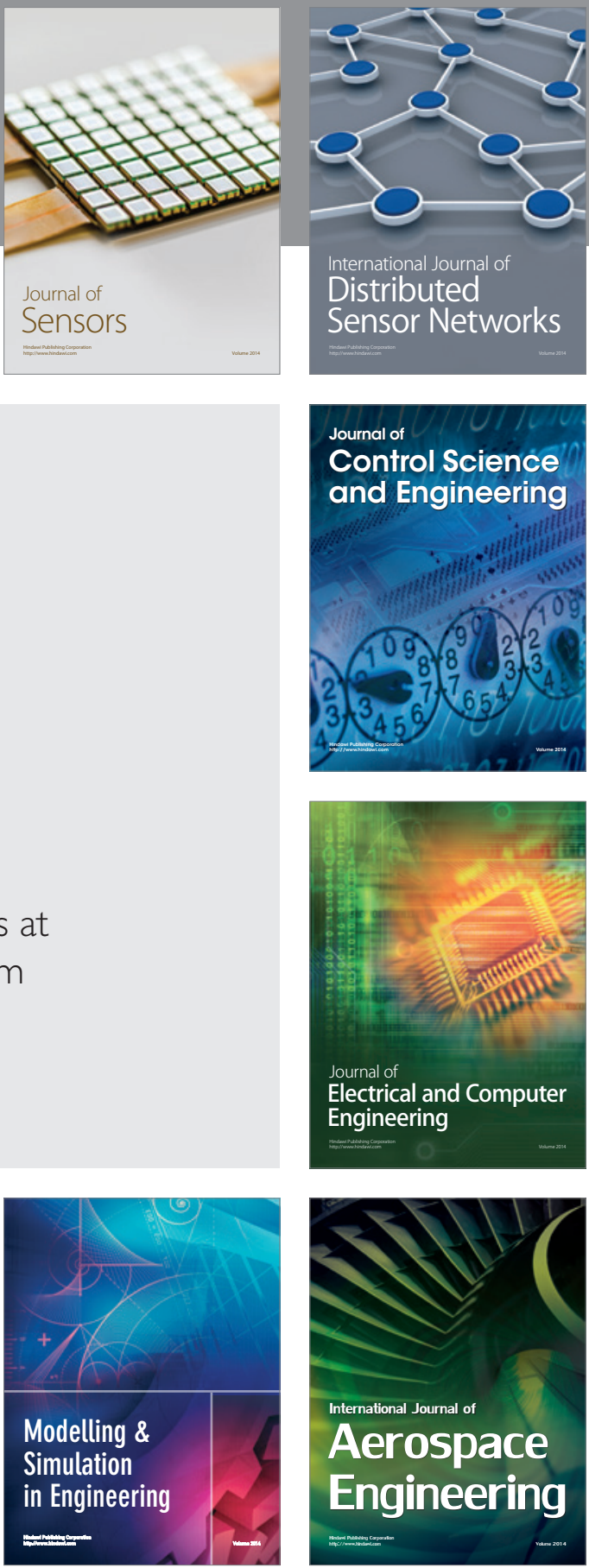

Journal of

Control Science

and Engineering
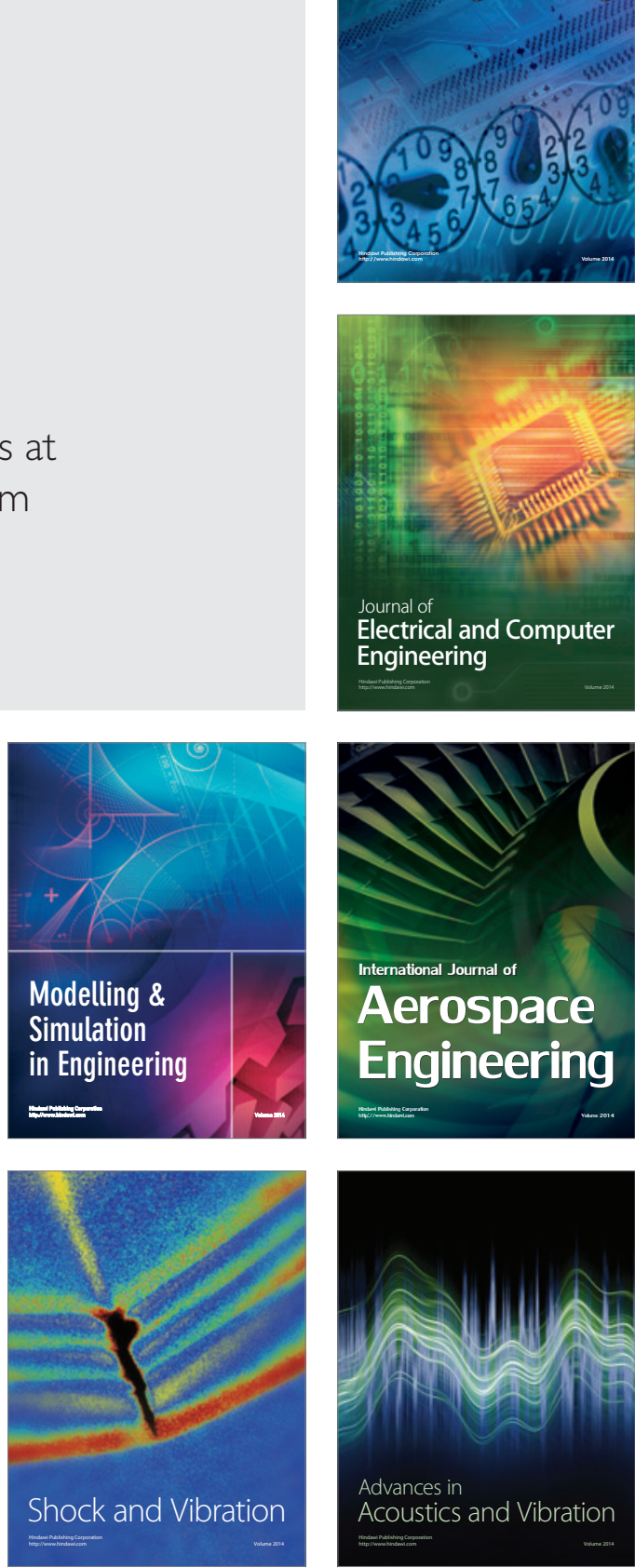